\title{
Testing the local spacetime dynamics by heliospheric radiocommunication methods
}

\author{
H.-J. Fahr and M. Siewert \\ Argelander-Institut für Astronomie, Abt. Astrophysik, Auf dem Hügel 71, 53121 Bonn, Germany \\ Received: 24 January 2008 - Revised: 17 March 2008 - Accepted: 31 March 2008 - Published: 13 May 2008
}

\begin{abstract}
According to general relativistic theories, cosmological spacetime is dynamic. This prediction is in excellent agreement with the huge majority of astronomical observations on large cosmic scales, especially the observations of cosmological redshifts of distant galaxies. However, on scales of heliospheric distances, verifications of general relativistic effects are based on Schwarzschild metric tests or kinetical corrections, such as the perihelion motion of Mercury, photon deflection at the Sun and gravitational photon redshifts in central gravity fields. As we will show in this paper, there is, however, a chance to detect new cosmologically relevant features on heliospheric scales by careful study of photon propagations in the local spacetime metrics, based on red- or blueshifts as a clear, but up to now overlooked, signature of the local spacetime dynamics. Thus, we propose the challenging possibility of carrying out experiments of cosmological relevance by simply using high-precision radio tracking of heliospheric spaceprobes, as already practised in cases like Pioneer-10/11, Galileo and Ulysses.
\end{abstract}

Keywords. Interplanetary physics (General or miscellaneous) - Radio science (Radio wave propagation; Space and satellite communication)

\section{Introduction}

When radiotracking distant spaceprobes from Earth, one may sooner or later ask the fundamental question, how the freely propagating radiophotons used to trace these objects, as well as the motion of these objects themselves, are affected by the dynamical metrical properties of spacetime that they cross on their geodetics. A partial answer to these questions is found in measurements of the gravitational redshift of photons, which provides a fundamental test of Einstein's general

Correspondence to: H. J. Fahr

(hfahr@astro.uni-bonn.de) relativistic equivalence principle, and which is the basis of all metric theories of gravity. According to this principle, clocks or oscillators should work locally Lorentz-invariant and position-invariant with respect to the gravitational potential. Due to its fundamental relevance this expectation has meanwhile been carefully tested by monitoring the frequency of a radiosignal transmitted by a stable oscillator when it moves in the gravitational field of a massive body like the sun or the planets (see Will, 1981; Anderson, 1989; Krisher, 1991). As reported by Krisher et al. (1990), the expected gravitational redshifts due to the gravity field of Saturn were registered at Voyager's flyby at Saturn. Furthermore, as published by Krisher et al. (1993), the predicted gravitational redshifts in the gravity field of the sun could in fact be verified up to $1 \%$ accuracy by the Galileo redshift experiment. Another test of the general relativistic perturbation of local space is measuring the Lense-Thirring effect (see Lense and Thirring, 1918), for which an experimental method has been proposed by Ciufolini (1986) and observationally carried out by Bertotti et al. (1987) who analysed lunar-LASER-ranging data to observe the geodetic lunar perigee precession and found this precession velocity to be within an accuracy of $10 \%$ to the predicted value of $19.2 \mathrm{~ms} /$ year (Mueller et al., 1991). However, observations of general relativistic spacetime perturbations are not restricted to astrophysical experiments, as demonstrated by Pound and Rebka (1959).

Despite an increasing number of successful general relativistic tests in the solar system, at least one unknown parameter remains in the model. Up to these days, no satisfying connection exists between the global cosmological Robertson-Walker metric describing the expanding universe and gravitationally bound, inhomogeneous systems, such as the solar system. This must provoke the question what kind of frequency shift photons experience on intermediate scales, i.e. scales small with respect to cosmic scales, but large with respect to Schwarzschild scales associated with pointlike masses. Depending on the distance from the individual 
central masses where cosmological effects start to contribute, it is quite possible that additional, previously unmodeled frequency shifts emerge in the solar system. And even if a (pseudo-)cosmological contribution to spacetime on these scales would not exist, one would still obtain a dynamic correction to the stationary Schwarzschild metric following from the general relativistic $N$-body problem. As demonstrated by several recent studies (see e.g. Fahr and Siewert, 2006, 2007; Carrera and Giulini, 2006; Bertolami et al., 2007 , and references therein), any reasonable form of a general relativistic description of local spacetime dynamics (i.e. comparable to the galactic spacetime) does not conflict with the classical, non-cosmological (or even non-relativistical) motions of planets around the sun, leaving only freely propagating (radio-)photons as a tool to measure the dynamical effects of spacetime. If, on the other hand, one could prove that cosmology already starts becoming important on scales of a few $\mathrm{AU}$, then the heliosphere would appear as an ideal and unique cosmic laboratory to test local features of cosmic spacetime by carrying out simple radiophoton communications in the heliosphere (see e.g. Masreliez, 2004; Rosales and Sanchez-Gomez, 1999; Carrera and Giulini, 2006; Petry, 2005; Scholz, 2007; Fahr and Siewert, 2006, 2007; Bertolami and Paramos, 2007) focusing on various sources of propagational photon frequency shifts, such as direct rescaling of the time intervals, recalibrations or dynamical rescaling effects. Considering the high complexity of the Einstein field equations, any detailed analysis of such effects would be very convoluted, and probably require numerical methods; for this reason, we instead try to give an answer to this question investigating alternatives to presently available approaches by introduction of a scale-dependent Robertson-Walker-like dynamic local scale function $L(t)$. We discuss general and special solutions for the temporal evolution of such local scale functions for different cosmic mass systems and identify one which for instance is able to explain the anomalous radiofrequency blueshift observed in the radiotracking data of the distant spacecrafts PIONEER-10 and -11 (see Anderson et al., 1998, 2002). In the next section, we derive this modified spacetime evolution on local scales.

\section{A sketch of modified spacetime evolutions on local scales}

First of all, in this context, it is important to notice that the average mass density in the universe is decreasing with the scale of the volume from which this average is taken. For instance, it falls off from the center of the solar system, where the mass density is mostly determined by the solar mass divided by volume, to the lowest level of the cosmic mass density which presently is expected at a value of about $\langle\rho\rangle_{L \rightarrow \infty} \simeq 10^{-31} \mathrm{~g} / \mathrm{cm}^{3}$ when averaged over so-called cosmological length scales. Systems of gravitationally bound masses with a total mass $M(L)$ thus have an average mass density $\rho(L)$ dependent on the time-dependent evolution of the associated scale $L=L(t, M)$ of the boundary shell of this mass system.

In the following, we describe the local spacetime contraction underlying the galactic structure formation process which begins just after the recombination point of cosmic matter at $t=t_{\text {rec }}$. Immediately after this time, cosmic matter can begin to contract on some critical scales $L_{\text {rec }}$ in the perturbed gravitational fields of the local mass density perturbations toward the centers of these perturbed regions.

As we have shown elsewhere (Fahr and Siewert, 2008), this suggests the use of the following differential equation to describe the motion of a test mass just at the border of this unstable region,

$\ddot{L}(t)=-\frac{G M}{L^{2}(t)}+L(t) \frac{\ddot{R}(t)}{R(t)}$,

where the first term is the usual force term due to a "central mass" of $M$, while the second term is the geodetic acceleration term derived by Cooperstock et al. (1999) where $R(t)$ is the scale of the universe and dots on top of the quantities denote derivatives with respect to time.

The above second-order differential equation can be converted into a first-order one of the following form (for details see Fahr and Siewert, 2008)

$\dot{L}^{2}=-\frac{2 G M}{L_{\mathrm{rec}}}+\frac{1}{2}\left(\frac{\ddot{R}}{R}+\frac{\ddot{R}_{\mathrm{rec}}}{R_{\mathrm{rec}}}\right) L^{2}+\dot{L}_{\mathrm{rec}}^{2}$.

For the further analysis we use the fact that, in a matterdominated universe, the conventional Friedmann equations lead to the well-known following relations (see e.g. Goenner, 1997)

$R(t)=R_{\mathrm{rec}}\left(\frac{t}{t_{\mathrm{rec}}}\right)^{2 / 3}$

$\dot{R}(t)=\frac{2}{3}\left(\frac{R(t)}{t_{\text {rec }}}\right)\left(\frac{t}{t_{\text {rec }}}\right)^{-1}=\frac{2}{3} \frac{R(t)}{t}$

$\ddot{R}(t)=-\frac{2}{9}\left(\frac{R(t)}{t_{\mathrm{rec}}^{2}}\right)\left(\frac{t}{t_{\mathrm{rec}}}\right)^{-2}=-\frac{2}{9} \frac{R(t)}{t^{2}}$.

Introduction of these relations then permits to derive from Eq. (2) the following equation:

$\left(\frac{\dot{L}}{L}\right)^{2}=\frac{\alpha}{L^{3}(t)}+\frac{\beta(t)}{L^{2}(t)}+\gamma(t)$,

with the introduction of

$\alpha=2 G M$ 
$\beta(t)=\left(\frac{2}{3 t_{\mathrm{rec}}} L_{\mathrm{rec}}\right)^{2}-\frac{2 G M}{L_{\mathrm{rec}}}+L_{\mathrm{rec}}^{2} \frac{1}{9 t_{\mathrm{rec}}^{2}}\left(1+\frac{t_{\mathrm{rec}}^{2}}{t^{2}}\right)$

$\gamma(t)=-\frac{1}{9 t_{\mathrm{rec}}^{2}}\left(1+\frac{t_{\mathrm{rec}}^{2}}{t^{2}}\right)$.

When considering the explicit time-dependence of the functions $\beta(t)$ and $\gamma(t)$, it becomes obvious that these functions should have become constants at the present-time of the universe, i.e. at $t \gg t_{\text {rec }}$. In this asymptotic form, the above equation reminds to the well known Friedmann-Lemàitre equation (see e.g. Blome et al., 2001, Eq. 6.41). Hereby the term $\alpha / L^{3}$ with its scale-dependence corresponds to the mass density term, while the term $\beta(t) / L^{2}$ corresponds to the curvature term with an asymptotic curvature parameter given by

$k_{\infty}=\beta(t \rightarrow \infty)=5 L_{\mathrm{rec}}^{2} / 9 t_{\mathrm{rec}}^{2}-2 G M / L_{\mathrm{rec}}$,

This points to the interesting fact that, on local scales $L$ local density perturbations $\rho(L) \geq\langle\rho\rangle_{L \rightarrow \infty}$ cause a nonvanishing, asymptotically constant spacetime curvature which, dependent on boundary conditions, either is positive or negative. Finally, the third term, $\gamma(t)$, in cosmological contexts is connected with the much discussed cosmological constant $\Lambda$, or the vacuum energy term. Here this term is always negative while in cosmological contexts it is mostly considered to be positive.

In the following we shall derive a particular solution for the case $L \gg L_{\text {rec }}$. Taking the asymptotic form of Eq. (2) for $L \gg L_{\text {rec }}$ one obtains

$\dot{L}^{2}=\left(\dot{L}_{\mathrm{rec}}^{2}-\frac{2 G M}{L_{\mathrm{rec}}}\right)+\frac{1}{2}\left(\frac{\ddot{R}}{R}+\frac{\ddot{R}_{\mathrm{rec}}}{R_{\mathrm{rec}}}\right) L^{2}$

and one notes that the first term on the RHS of this equation is fixed by the initial cosmic conditions at $t=t_{\text {rec }}$. Adopting as initial condition at the recombination phase the following relation

$C_{\text {rec }}=\left(\dot{L}_{\text {rec }}^{2}-\frac{2 G M}{L_{\text {rec }}}\right)=0$

one then obtains

$\frac{\dot{L}}{L}= \pm \sqrt{\frac{1}{2}\left(\frac{\ddot{R}}{R}+\frac{\ddot{R}_{\mathrm{rec}}}{R_{\text {rec }}}\right)}$,

where the " + " -sign has to be used for times with $t \leq t_{0}$, and the "-"-sign for times $t \geq t_{0}$, with the time $t_{0}$ defined by $\dot{L}_{0}=\dot{L}\left(t_{0}\right)=0$. This then leads to the solution

$L=L_{0} \exp \left[-\int_{t_{0}}^{t} \sqrt{\frac{1}{2}\left(\frac{\ddot{R}}{R}+\frac{\ddot{R}_{\mathrm{rec}}}{R_{\mathrm{rec}}}\right)} d t\right]$

where $L_{0}=L\left(t_{0}\right)$ is determined by the condition $\dot{L}\left(L_{0}\right)=0$ and $\dot{L} \leq 0$ for $t \geq t_{0}$. The above relation is acceptable as long as $L(t) \gg L_{\text {rec }}$.

\section{Frequency shifts of freely propagating photons}

In the following we study the frequency shift of a radiophoton freely propagating in a locally modified RobertsonWalker spacetime. The scale of the latter is assumed to be associated with a local scale factor $L(t)$, which does not follow the same law valid for the global RW scale factor $R(t)$, as we have already discussed above. If the time-dependent, local scale factor $L(t)$ governs the metric in the solar system, we may make use of a local variant of the cosmological wavelength-redshift relation (see e.g. Goenner, 1997, p. 443), and are then lead to the relation

$\frac{\lambda_{1}}{\lambda_{0}}=\frac{\nu_{0}}{\nu_{1}}=\frac{L_{1}}{L_{0}}$,

The subscript 0 denotes the respective value of the emitted photon, while the subscript 1 denotes the observed, shifted photon after a free passage time $2 t_{i}$, with $t_{i}$ being the oneway passage time of a radio signal from Earth to a distant spaceprobe. Here we have called the initial scale factor $L(t=0)=L_{0}$, while the scale factor at $t=2 t_{i}$ is called $L_{1}$. For short time intervals (i.e. $2 t_{i} \ll 1 / H_{0}$ ), it is possible to use the Taylor expansion $L_{1}=L_{0}+\dot{L}_{0} \cdot 2 t_{i}$, and one obtains

$\Delta v=v_{0}-v_{1}=v_{0}\left(1-\frac{L_{0}}{L_{0}+\dot{L}_{0} 2 t_{i}}\right)=v_{0} \frac{\dot{L}_{0}}{L_{0}} 2 t_{i}$.

Depending on the expansion or contraction rate of the local spacetime scale $L(t)$, and the base frequency and propagation time of the radiophotons, this relation may also be applied to estimate how far the spacecraft has to go out from the observer for the effect to be observable, by setting $\Delta v>\Delta v_{\text {obs }}$, where $\Delta v_{\text {obs }}$ is the absolute accuracy obtainable by the experiment. Considering that $t_{i} \approx x_{i} / c$, we obtain

$\frac{\dot{L}}{L}>2.5 \frac{\Delta v_{\mathrm{obs}}}{v_{0}}\left(\frac{x}{1 \mathrm{AU}}\right)$,

which allows us to estimate an upper limit for the sensitivity. If we assume that the spacetime expansion or contraction rate is of the order of the present day Hubble constant, i.e. $H_{0}=o\left(10^{-12}\right) \mathrm{s}^{-1}$, we obtain the requirement

$10^{-12}>\frac{\Delta v_{o b s}}{v_{0}}\left(\frac{x}{1 \mathrm{AU}}\right)$,

which means that, effectively, the required experimental precision scales linearly with the length of the photon path. Nevertheless, the measurement of the radiofrequency must be very precise, which, however, nowadays is well possible using modern radio communication methods with highly stable oscillators (see Anderson et al., 2002).

\section{Conclusions}

In the section above we have shown that, in the framework of a simplified theory of heliospheric spacetime dynamics, the 
spacetime geometry is characterized by a typical scale function $L=L(M, t)$ with a time-dependence given by Eq. (2). In case of a special initial condition $C_{\mathrm{rec}}=\left(\dot{L}_{\mathrm{rec}}^{2}-\frac{2 G M}{L_{\mathrm{rec}}}\right)=0$, one obtains the solution in the form of Eq. (16)

$$
\dot{L} / L=-H_{\Lambda},
$$

yielding an additional, previously unmodeled frequency shift in the radiotracking signal of heliospheric spacecrafts of

$\Delta v=v_{0} \frac{\dot{L}_{0}}{L_{0}} 2 t_{i}=-H_{\Lambda} v_{0} 2 t_{i}$

which is just the result needed to quantitatively explain the PIONEER blueshift anomaly (see Anderson et al., 2002). This does not prove that with the above we have found the correct explanation of this anomaly, but it may point to a completely new kind of experiments, using similar radiotracking observations for the sake of determining the local spacetime metric. In addition, direct observations of such metrically-induced redshifts might allow to measure, for the first time, the full energy-stress tensor generating our spacetime, including contributions from the dark side of the universe (i.e. dark matter and dark energy).

Even in face of a considerably more complicated heliospheric spacetime than the simple model considered here, which must be expected in an $N$-body system, the general idea of frequency shifts on account of a specific dynamical behaviour of the spacetime along the photon path (instead of a simple special-relativistic Doppler-shift) is firmly rooted in the theory of general relativity and commonly applied in cosmological studies. However, the question whether this effect also appears on heliospheric scales remains unanswered, and can likely only be solved using experimental methods.

Acknowledgements. Topical Editor R. Forsyth thanks S. Asmar for his help in evaluating this paper.

\section{References}

Anderson, J. D.: Gravitational experiments on a solar probe mission: Scientific objectives and technology considerations, in: Relativistic Gravitational Experiments in Space, vol. 3046 of NASA Conference Proceedings, pp. 148-154, 1989.

Anderson, J. D., Laing, P. A., Lau, E. L., Liu, A. S., Nieto, M. M., and Turyshev, S. G.: Indication, from Pioneer 10/11, Galileo, and Ulysses Data, of an Apparent Anomalous, Weak, Long-Range Acceleration, Phys. Rev. Lett., 81, 2858-2861, 1998.

Anderson, J. D., Laing, P. A., Lau, E. L., Liu, A. S., Nieto, M. M., and Turyshev, S. G.: Study of the anomalous acceleration of Pioneer 10 and 11, Phys. Rev. D, 65, 082004 , for an updated version see www.arxiv.org/abs/gr-qc/0104064, 2002.

Bertolami, O. and Paramos, J.: A Mission to test the Pioneer anomaly: Estimating the main systematic effects, www.arxiv. org/abs/gr-qc/0702149, 2007.
Bertolami, O., Böhmer, C. G., Harko, T., and Lobo, F. S. N.: Extra force in $f(R)$ modified theories of gravity, www.arxiv.org/abs/ 0704.1733, 2007.

Bertotti, B., Ciufolini, I., and Bender, P. L.: New test of general relativity: Measurement of de Sitter geodetic precession rate for lunar perigee, Phys. Rev. Lett., 58, 1062-1065, 1987.

Blome, H. J., Hoell, J., and Priester, W.: Kosmologie, vol. 8 of Lehrbuch der Experimentalphysik, Berlin, W. de Gruyter, 2001.

Carrera, M. and Giulini, D.: On the influence of the global cosmological expansion on the local dynamics of the solar system, www.arxiv.org/abs/gr-qc/0602098, 2006.

Ciufolini, I.: Measurement of the Lense-Thirring drag on highaltitude, laser-ranged artificial satellites, Phys. Rev. Lett., 56, 278-281, 1986.

Cooperstock, F. I., Faraoni, V., and Vollick, D. N.: The influence of the cosmological expansion on local systems, Astrophys. J., 501, 61-66, 1999.

Fahr, H.-J. and Siewert, M.: Does Pioneer measure local spacetime expansion?, www.arxiv.org/abs/gr-qc/0610034, 2006.

Fahr, H.-J. and Siewert, M.: Local Spacetime Dynamics, the Einstein-Straus Vacuole and the PIONEER Anomaly: a New Access to these Problems, Z. Naturforsch. A, 62, 1-10, 2007.

Fahr, H.-J. and Siewert, M.: Imprints from the global cosmological expansion to the local spacetime dynamics, Naturwissenschaften, doi:10.1007/s00114-007-0340-1, 2008.

Goenner, H.: Einführung in die Kosmologie, Heidelberg: Spektrum Akademischer Verlag, 1997.

Krisher, T. P.: New tests of the gravitational redshift effect, Mod. Phys. Lett. A, 5, 1809-1813, 1991.

Krisher, T. P., Anderson, J. D., and Campbell, J. K.: Test of the gravitational redshift effect at Saturn, Phys. Rev. Lett., 64, 13221325, 1990.

Krisher, T. P., Morabito, D. D., and Anderson, J. D.: The Galileo solar redshift experiment, Phys. Rev. Lett., 70(15), 2213-2216, 1993.

Lense, J. and Thirring, H.: Über den Einfluss der Eigenrotation der Zentralkörper auf die Bewegung der Planeten und Monde nach der Einsteinschen Gravitationstheorie, Phys. Z., 19, 156163, translation in General Relativity and Gravitation, 16(1984), 727-741, 1918.

Masreliez, C. J.: Scale Expanding Cosmos Theory I - An introduction, Apeiron, 11, 99-133, http://redshift.vif.com, 2004.

Mueller, J., Schneider, M., Soffel, M., and Ruder, H.: Testing Einstein's theory of gravity by analyzing Lunar Laser Ranging data, Astrophys. J. Lett., 382, L101-L103, doi:10.1086/186222, 1991.

Petry, W.: Further Results of Flat Space-time Theory of Gravitation, Z. Naturforsch., 60a, 255-264, 2005.

Pound, R. V. and Rebka, G. A.: Gravitation Red-Shift in Nuclear Resonance, Phys. Rev. Lett., 3, 439-441, 1959.

Rosales, J. L. and Sanchez-Gomez, J. L.: The "Pioneer effect" as a manifestation of the cosmic expansion in the solar system, www. arxiv.org/abs/gr-qc/9810085, 1999.

Scholz, E.: Another look at the Pioneer anomaly, www.arxiv.org/ abs/astro-ph/0701032, 2007.

Will, C. M.: Theory and Experiment in Gravitational Physics, Cambridge: Cambridge University Press, 1981. 